\title{
Expression Analysis of the First Arbuscular Mycorrhizal Fungi Aquaporin Described Reveals Concerted Gene Expression Between Salt-Stressed and Nonstressed Mycelium
}

\author{
Ricardo Aroca, ${ }^{1}$ Alberto Bago, ${ }^{1}$ Moira Sutka, ${ }^{2}$ José Antonio Paz, ${ }^{1}$ Custodia Cano, ${ }^{1}$ Gabriela Amodeo, ${ }^{2}$ \\ and Juan Manuel Ruiz-Lozano' \\ ${ }^{1}$ Departamento de Microbiología del Suelo y Sistemas Simbióticos, Estación Experimental del Zaidín, CSIC, Profesor \\ Albareda, 1, 18008, Granada, Spain; ${ }^{2}$ Laboratorio de Biomembranas, Fisiología y Biofísica, Facultad de Medicina, \\ Universidad de Buenos Aires, C1121ABG Buenos Aires, Argentina
}

Submitted 7 April 2009. Accepted 22 May 2009.

\begin{abstract}
Roots of most plants in nature are colonized by arbuscular mycorrhizal (AM) fungi. Among the beneficial effects of this symbiosis to the host plant is the transport of water by the AM mycelium from inaccessible soil water resources to host roots. Here, an aquaporin (water channel) gene from an AM fungus (Glomus intraradices), which was named GintAQP1, is reported for the first time. From experiments in different colonized host roots growing under several environmental conditions, it seems that GintAQP1 gene expression is regulated in a compensatory way regarding host root aquaporin expression. At the same time, from in vitro experiments, it was shown that a signaling communication between NaCl-treated mycelium and untreated mycelium took place in order to regulate gene expression of both GintAQP1 and host root aquaporins. This communication could be involved in the transport of water from osmotically favorable growing mycelium or host roots to salt-stressed tissues.
\end{abstract}

The colonization of dry land by aquatic plants approximately 400 million years ago was possible thanks to the establishment of a symbiosis between plants and fungi (Pirozynski and Malloch 1975), including arbuscular mycorrhizal (AM) fungi (Bonfante and Genre 2008). Thanks to this symbiosis, plants were able to colonize a stressful environment such as the dry land. Thus, both partners of the symbiosis co-evolved successfully to colonize almost all dry-land ecosystems, from the tropics to subpolar latitudes (Rosendahl 2008). In the AM symbiosis, plants get nutrients and water resources that are less available to the plant roots from the fungi, while the fungi receive carbon compounds from the plant and find a niche to complete their life cycle (Koide and Mosse 2004). At the same time, AM symbiosis enhances plant tolerance to different abiotic stresses with osmotic components such as drought and salinity (Augé 2001; Jahromi et al. 2008; Ruiz-Lozano 2003; Ruiz-Lozano et al. 2006).

Under osmotic stress conditions, plants need to finely regulate their water relations. Thus, when plants are exposed to an osmotic stress, the first symptoms are a diminution of their root water permeability and a decrease of their leaf transpiration rate (Aroca et al. 2006; Muhsin and Zwiazek 2002). By

Corresponding author: Ricardo Aroca; E-mail: raroca@eez.csic.es both mechanisms, plants try to save as much water as possible. However, the relationship between diminutions of leaf transpiration or water uptake capacity and tolerance to osmotic stresses is not well established. Under certain osmotic stress conditions, it can be helpful to the plant to increase its root hydraulic conductivity and then enhance its water uptake capacity. However, under other circumstances, it can be more efficient to reduce root hydraulic conductivity in order to avoid water flow from roots to the soil when the water potential of the soil decreases. These apparent contradictory behaviors are still a matter of debate (Maurel et al. 2008). However, most recently, Matsuo and associates (2009) found a positive correlation between shoot dry weight and root hydraulic conductivity under water-limited conditions in different rice genotypes.

It is commonly accepted that plant water relations are ultimately regulated by aquaporins activity (Forrest and Bhave 2007; Maurel et al. 2008). Aquaporins are proteinaceous pores present in the membranes of all living organisms that facilitate the transport of water and other small and neutral solutes, always following an osmotic gradient (Chrispeels and Agre 1994; Forrest and Bhave 2007; Maurel et al. 2008; Pettersson et al. 2005). In plants, aquaporins are part of a large and diverse protein family consisting of 31 to 37 different genes (Maurel et al. 2008; Sade et al. 2009) depending on the plant species. Therefore, plant aquaporins are subdivided into four groups based on their amino acid sequence homology: plasma membrane intrinsic proteins (PIP), tonoplast intrinsic proteins (TIP), nodulin-like intrinsic proteins (NIP), and small and basic intrinsic proteins (SIP). Most recently, a fifth plant aquaporin group has been described in tomato, and it was called XIP (Sade et al. 2009). The role of aquaporins in plant drought and salt tolerance is still uncertain, due to contradictory results. Thus, constitutive overexpression of PIP genes in tobacco and rice enhanced plant sensitivity to drought and salinity (Aharon et al. 2003; Katsuhara et al. 2003). However, when a PIP gene was overexpressed in rice under the control of an inducible stress promoter, an enhancement of drought avoidance was achieved (Lian et al. 2004). Most recently, Yu and associates (2005) found that tobacco plants overexpressing or silencing some PIP aquaporin genes were more sensitive and tolerant, respectively, to drought than the untransformed plants. At the vacuolar level (tonoplast), Peng and associates (2007) found that Arabidopsis plants overexpressing a Panax ginseng TIP gene were more tolerant to salt stress but less tolerant to 
drought. On the other hand, tomato plants overexpressing a TIP gene were more drought tolerant than wild-type plants (Sade et al. 2009). Moreover, the manipulation of the expression of one aquaporin gene also alters the expression pattern of the whole aquaporin gene family (Jang et al. 2007). All these data highlight the specific role of each aquaporin gene under different environmental stress conditions.

Roussel and associates (1997) and Krajinski and associates (2000) were the first authors reporting an alteration of aquaporin gene expression by AM symbiosis in parsley and Medicago truncatula roots, respectively. In both works, AM symbiosis caused an upregulation of a TIP gene. Most recently, a review dealing with this topic has been published (Uehlein et al. 2007). The above reports were carried out in the absence of any stress. However, during the last few years, evidence has arisen showing the regulation of plant aquaporins by AM symbiosis under osmotic stress conditions (Ruiz-Lozano and Aroca 2008; Ruiz-Lozano et al. 2006). Thus, Porcel and associates (2006a) found an anticipated downregulation of two PIP genes in soybean and lettuce roots inoculated with an AM fungus during a drought treatment compared with noninoculated plants. These results were interpreted as a mechanism of avoiding water flow from roots to soil while soil was drying progressively. Ouziad and associates (2006) also found similar results analyzing one PIP and one TIP gene in tomato roots subjected to salt stress. However, in Phaseolus vulgaris plants inoculated with the AM fungus Glomus intraradices subjected to salt stress, three of four PIP genes analyzed increased their root expression in a higher proportion than in noninoculated plants (Aroca et al. 2007). When the same plants were subjected to drought, the expression of each PIP gene responded in a different way (up- or downregulated) depending on the presence or absence of the AM fungus. The above data support the idea that each plant aquaporin has its specific function under each environmental stress condition (Jang et al. 2007). By contrast, although it is largely known that AM mycelium transports water from the soil to the host roots (Hardie 1985; Khalvati et al. 2005; Marualanda et al. 2003; Ruiz-Lozano and Azcón 1995), no information concerning the regulation of fungal aquaporins during different osmotic stresses is available.

In the fungal kingdom, five kinds of aquaporins have been described (Pettersson et al. 2005). They are subdivided into orthodox aquaporins (two groups), initially described in Saccharomyces cerevisiae (Laizé et al. 2000); three different groups of aquaglyceroporins, described in $S$. cerevisiae (Oliveira et al. 2003; Van Aelst et al. 1991); and filamentous fungi (Pettersson et al. 2005). Aquaglyceroporins are able to transport small solutes as glycerol, urea, and other small molecules, instead of or co-transported with water (Rojek et al. 2008; Wallace et al. 2006). However, no aquaporins have been described so far from any AM fungus. Here, we cloned for the first time a putative aquaporin gene from the AM fungus $G$. intraradices (GintAQP1) and studied its regulation under a range of osmotic stress conditions, hosts plants, and growing media (soil or in vitro). The results from the in vitro studies highlight the interplay regulation of GintAQP1 and plant host aquaporin genes during an osmotic stress episode.

\section{RESULTS}

Cloning of $G$. intraradices aquaporin cDNA (GintAQP1).

A cDNA was synthesized from total RNA extracted from $G$. intraradices hyphae growing in the hyphal compartment (HC) of a monoxenic culture (Porcel et al. 2006b; St.-Arnaud et al. 1996). By using degenerate primers, based on the NPA conserved motifs of several plants, yeasts, and filamentous fungi aquaporins, a partial clone of a putative aquaporin from
G. intraradices was obtained. Later, by the rapid amplification of cDNA ends (RACE) technique, we cloned the fulllength cDNA sequence of the $G$. intraradices aquaporin and called it GintAQP1 (accession number FJ861239). The coding region comprised 762 bp corresponding to 253 amino acids (Fig. 1), with an expected molecular weight of $27.2 \mathrm{kDa}$. The GintAQP1 has the two NPA motifs present in almost all aquaporins, and it also has the six transmembrane domains common to all aquaporins (Fig. 1) (Pettersson et al. 2005). Because the original biological material could be contaminated by carrot (Daucus carota L.) root residues (Recorbet et al. 2009), we extracted genomic DNA from $G$. intraradices spores, hyphae, and carrot roots and amplified the full open reading frame of GintAQP1 by polymerase chain reaction (PCR) with specific primers (Fig. 2A). PCR amplified a fragment of approximately 1,200 bp only in the spore and hyphae DNA but not in that from carrot roots. Then, we transferred the DNA from the agarose gel to a Hybond-N membrane and hybridized it with the GintAQP1 full open reading frame DNA as a probe. The signal was only detected at the same position as in the previous PCR (Fig. 2B). These data confirmed the fungal origin of the GintAQP1 gene.

After a phylogenetic analysis, GintAQPl was found to not be integrated in any specific cluster, indicating specific features among fungal aquaporins (Fig. 2C). However, GintAQP1 shares with endoplasmatic reticulum (ER) aquaporins a short $\mathrm{N}$ terminal without any diacid motif (Fig 1; Maeshima and Ishikawa 2008). At the same time, we carried out experiments with oocytes from Xenopus laevis in order to test the capacity of GintAQP1 protein to transport water, glycerol, or urea. The results showed no transport activity for any of the three compounds tested (data not shown). In contrast, the positive control used showed water, glycerol, or urea transport capacity, discarding any problem during the experimental procedures.

\section{GintAQP1 expression under different stress conditions and host plants.}

We tested the regulation of GintAQP1 gene expression by quantitative real-time PCR in roots of different host plants, under different stress conditions, and under hormonal treatment (Porcel et al. 2006b). First, we evaluated the regulation of GintAQP1 expression in $P$. vulgaris colonized roots under drought, cold, or salt stresses (Aroca et al. 2007). The expression of the GintAQP1 gene increased under drought and cold stresses but remained constant under salt stress (Fig. 3A). No significant differences in the percentage of mycorrhizal root length (percentage of root length that containing fungal structures) were observed among different stress treatments (Aroca et al. 2007). Later, we analyzed the expression of the GintAQP1 gene in colonized roots of lettuce (Lactuca sativa L.) under well-watered conditions or subjected to drought and treated or not with abscisic acid (ABA) (Aroca et al. 2008), a plant hormone that regulates stomatal closure and root hydraulic properties (Hartung et al. 2005). GintAQP1 gene expression was decreased dramatically by both drought and ABA treatments, being undetectable when both treatments were combined (Fig. $3 \mathrm{~B}$ ), even if the percentage of mycorrhizal root length was increased (Aroca et al. 2008). Finally, we evaluated GintAQP1 expression in colonized roots of two different lines of tobacco (Nicotiana tabacum L.) subjected to drought stress (Porcel et al. 2005): a wild-type and antisense line where a PIP1 gene and its related aquaporin genes have reduced their expression by approximately $80 \%$ (Siefritz et al. 2002). The only significant difference observed between the two lines on the expression of GintAQP1 was found under drought conditions, where the antisense line showed higher expression levels than the wild-type one (Fig. 3C). 
GintAQP1 expression in monoxenic culture.

One of the most extended methods to study the regulation of AM fungi genes in the extraradical mycelium is the use of the monoxenic culture (Allen and Shachar-Hill 2009; Porcel et al. 2006b; Recobert et al. 2009). Here, we intended to ascertain how the GintAQP1 gene was regulated in the extraradical my- celium under environmental conditions known to regulate plant aquaporins expression. Therefore, we added to the HC ABA polyethylene glycol as an osmotic agent or $\mathrm{NaCl}$. We also incubated other plates at $5^{\circ} \mathrm{C}$ for 3 days. No changes in GintAQP1 gene expression levels in the extraradical mycelium were observed for any of these treatments (data not shown).

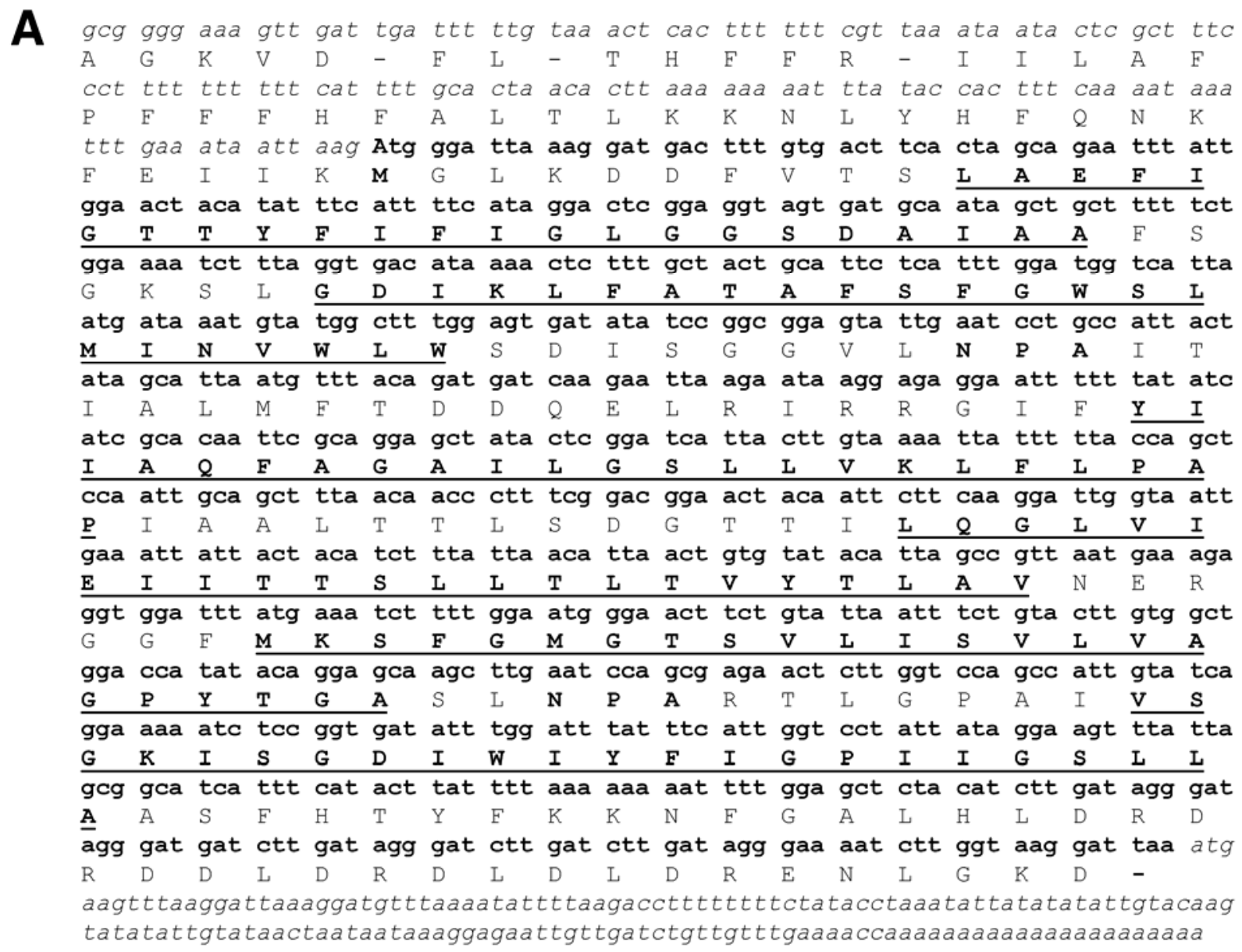

B

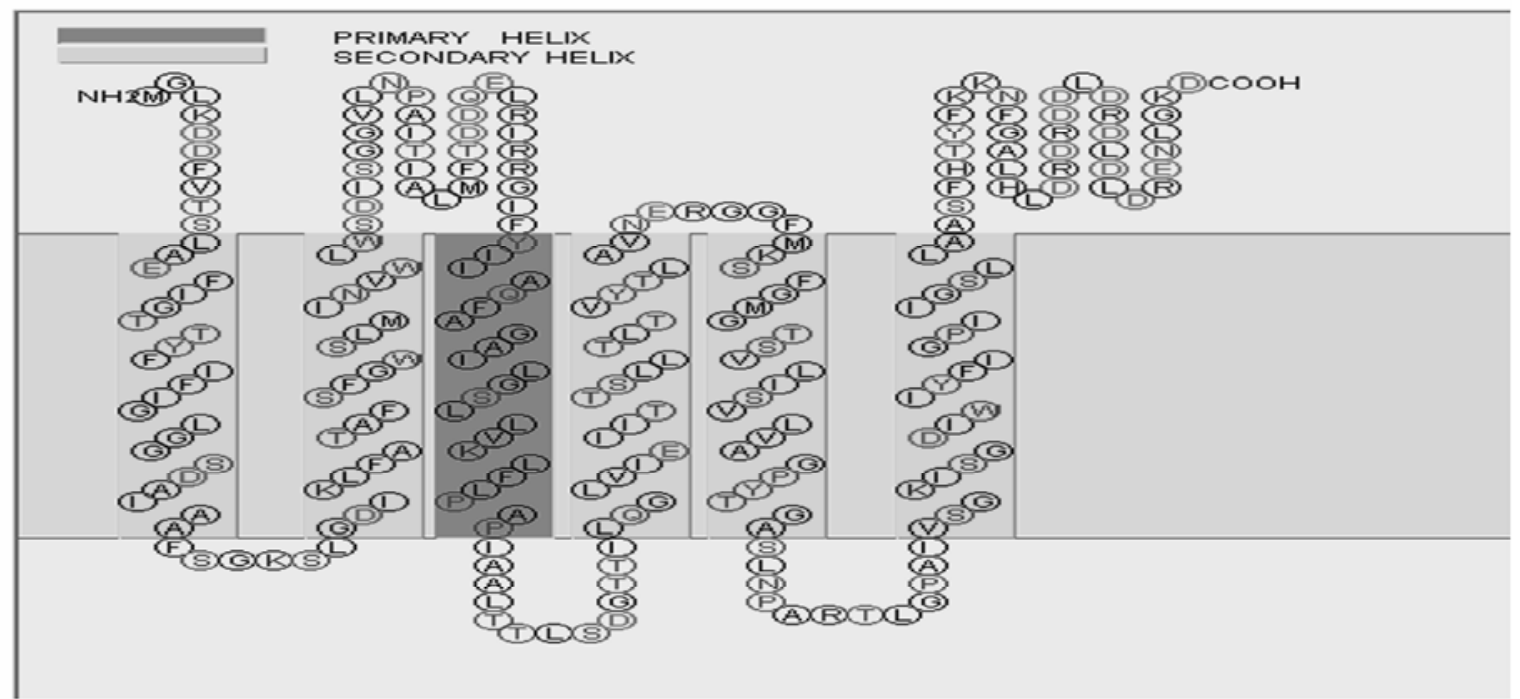

Fig. 1. A, Nucleotide and amino acid sequence of the GintAQP1 gene, including $5^{\prime}$ and $3^{\prime}$ untranslated regions. The first methionine is marked in bold, as it is the two NPA motifs characteristic of aquaporin genes. The six transmembrane domains are in bold and underlined. Note the short N-terminal region composed of only 10 amino acids. B, Picture of the hypothetical transmembrane domains generated by the SOSUI program (Hirokawa et al. 1998). 

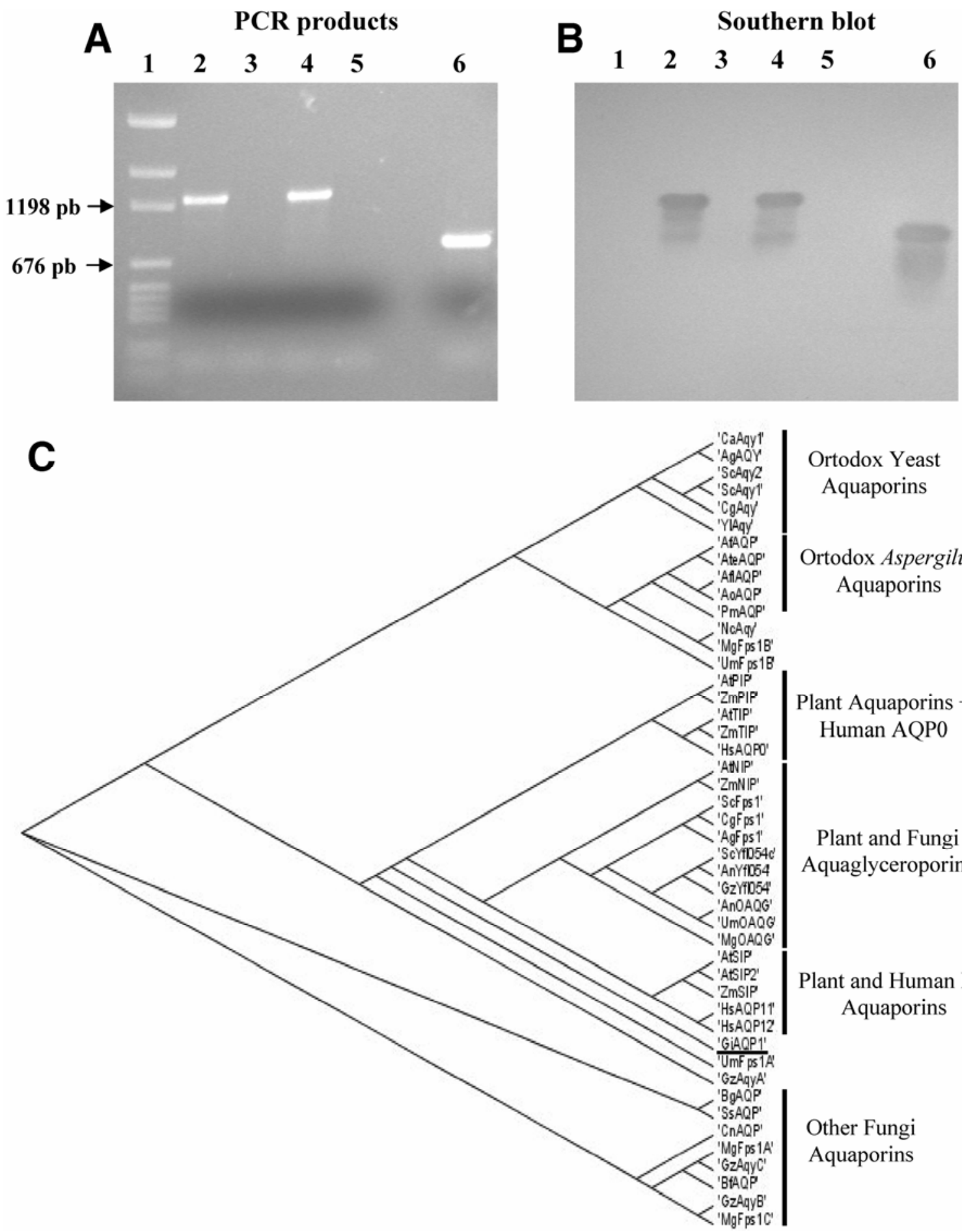

Ortodox Yeast

Aquaporins

Ortodox Aspergilus Aquaporins

Plant Aquaporins + Human AQP0

Plant and Fungi Aquaglyceroporins

Plant and Human ER Aquaporins

Fig. 2. A, Electrophoresis on $1.2 \%$ agarose gel for polymerase chain reaction (PCR) products obtained with specific primers designed on the GintAQP1 gene B, Southern blot hybridization using the full open reading frame (ORF) of the GintAQP1 as a probe. Lane 1, Molecular weight marker; 2, genomic DNA from Glomus intraradices spore; 3, genomic DNA from Daucus carota root; 4, genomic DNA from G. intraradices mycelium; 5, negative control (DNA-free water); 6, positive control (pGEM plasmid carrying the ORF cDNA of GintAQP1). C, Phylogenetic tree of various human, plant, and fungi aquaporins, including GintAQP1 (underlined), using neighbor-joining method. The abbreviations for species names are Ca (Candida albicans), Ag (Ashbya gossypii), Sc (Saccharomyces cerevisiae), Cg (Candida glabrata), Yl (Yarrowia lipolytica), Af (Aspergillus fumigatus), Ate (Aspergillus terreus), Afl (Aspergillus flavus), Ao (Aspergillus oryzae), Pm (Penicillium marnefei), Nc (Neurospora crassa), Mg (Magnaporthe grisea), Um (Ustilago maydis), At (Arabidopsis thaliana), $\mathrm{Zm}$ (Zea mays), Hs (Homo sapiens), An (Aspergillus nidulans), Gi (Glomus intraradices), Gz (Gibberella zeae), Bg (Blumeria graminis), Ss (Sclerotinia sclerotiorum), Cn (Cryptococcus neoformans), and $\mathrm{Bf}$ (Botryotinia fuckeliana). The accession numbers of the amino acid sequences used are CaAqy1 (EAK96766), AgAQY (AAS54225), ScAqy2 (AAD10058), ScAqy1 (BAG50026), CgAqy1 (CAG57715), YlAqy (CAG77656), AfAQP (EDP47366), AteAQP (EAU37108), AflAQP (EED52460), AoAQP (BAE64845), PmAQP (EEA19904), NcAqy (EAA35947), MgFps1B (EDJ99463), UmFps1B (EAK84000), AtPIP (CAB71073), ZmPIP (X82633), AtTIP (AAD31569), ZmTIP (AF037061), HsAQP0 (AAH74913), AtNIP (CAA16748), ZmNIP (AF326483), ScFps1 (CAA38096), CgFps1 (CAG58224), AgFps1 (AAS51160), ScYfl054c (AAT92622), AnYfl054 (EAA63393), GzYfl054 (EAA71472), AnOAQG (EAA59224), UmOAQG (EAK82563), MgOAQG (EDJ96530), AtSIP (AAF26804), AtSIP2 (CAB72165), ZmSIP (AF326497), HsAQP11 (EAW75031), HsAQP12 (BAC45006), GiAQP1 (FJ861239), UmFps1A (EAK81040), GzAqyA (EAA70757), BgAQP (CAD66431), SsAQP (EDN98188), CnAQP (AAW42227), MgFps1A (EDJ99173), GzAqyC (EAA75170), BfAQP (EDN26017), GzAqyB (EAA71493), and MgFps1C (EDJ94979). 
Therefore, we analyzed how GintAQP1 gene expression was regulated in the root compartment (RC), mainly composed of colonized carrot roots together with small amount of extraradical mycelium, when $\mathrm{NaCl}$ was added to the $\mathrm{HC}$. Curiously, the expression of GintAQP1 was higher in $\mathrm{RC}$ than in $\mathrm{HC}$ under control conditions but, more interestingly, the expression increased in the RC compartment upon exposing $\mathrm{HC}$ to $100 \mathrm{mM}$ $\mathrm{NaCl}$ (Fig. 4). In a second experiment, we added the same concentration of $\mathrm{NaCl}$ to the $\mathrm{RC}$ and checked the GintAQP1 ex- pression in both compartments. Unexpectedly, GintAQP1 expression profusely rose in the $\mathrm{HC}$ and only marginally and nonsignificantly in the RC (Fig. 4).

\section{Carrot aquaporins regulation under salt stress.}

From the previous data, we grew interested in knowing how carrot aquaporins could be regulated by the AM symbiosis as well as by salt stress applied in both plate compartments. Thus, using the same methods and degenerate primers described for
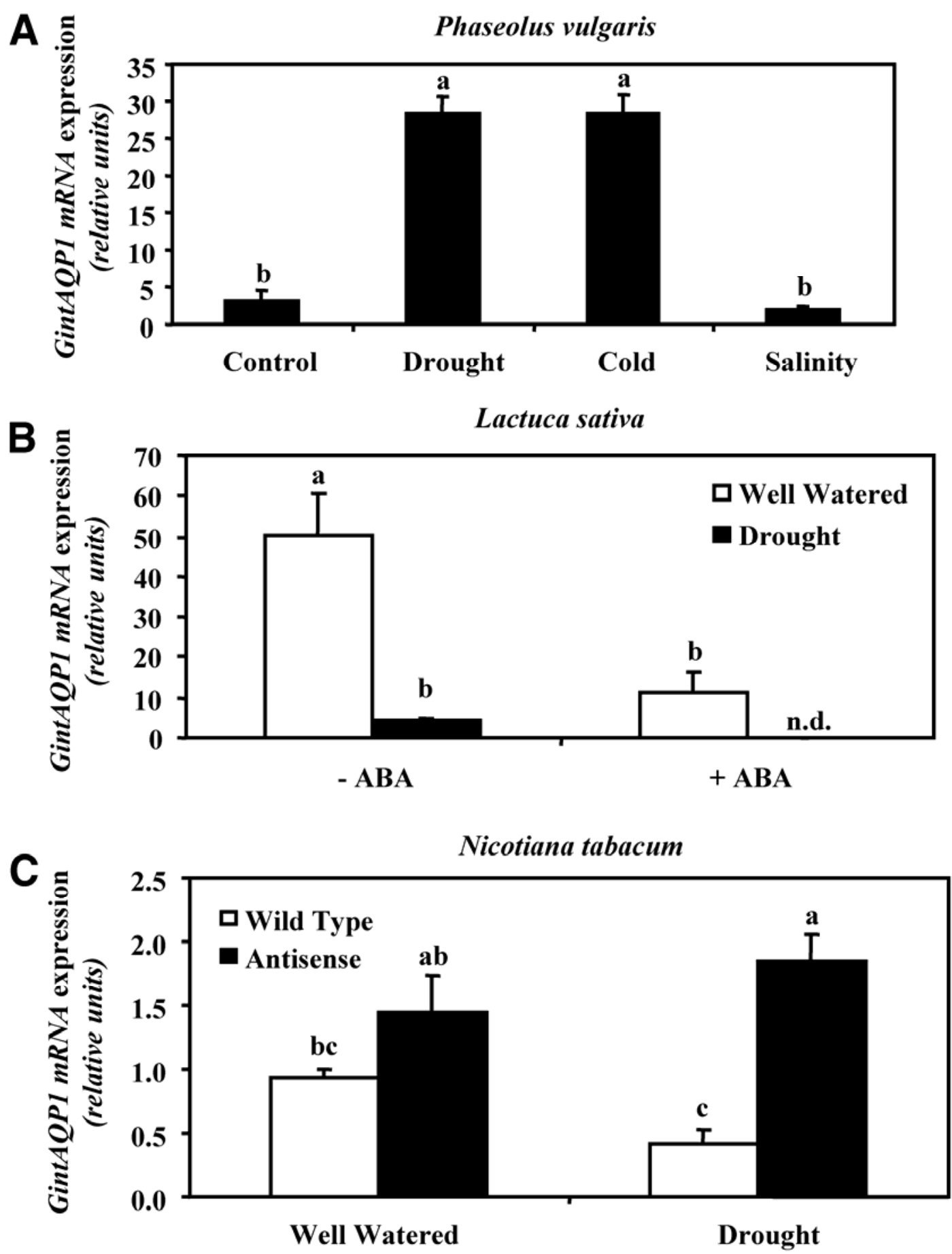

Fig. 3. GintAQP1 gene expression analyses in several host roots and environmental conditions. A, GintAQP1 gene expression in Phaseolus vulgaris roots colonized by Glomus intraradices untreated (Control) without watering for 4 days (Drought), subjected to $4^{\circ} \mathrm{C}$ for 2 days (Cold), and subjected to $60 \mathrm{mM}$ $\mathrm{NaCl}$ (Salinity). B, GintAQP1 gene expression in Lactuca sativa roots colonized by G. intraradices and cultivated under well-watered conditions (white bars) or grown under $70 \%$ soil-water-holding capacity for 10 days (Drought, black bars), treated or not with $100 \mu \mathrm{M}$ abscisic acid $(\mathrm{ABA})$; n.d. $=$ not detected. C, GintAQP1 gene expression in Nicotiana tabacum wild-type (white bars) or NtAQP1 antisense (black bars) roots colonized by G. intraradices and cultivated under well-watered conditions or under $70 \%$ soil-water-holding capacity for 10 days (Drought). Bars represent mean \pm standard error $(n=6)$. Bars with the same letter indicate nonsignificant differences $(P>0.05)$ after analysis of variance and least significant difference tests. 
the cloning of the GintAQP1 gene and RNA extracted from carrot roots growing in vitro without any AM fungus, we obtained two different clones of putative carrot aquaporins. After RACE experiments, we obtained the full-length cDNAs of the two clones and called them DcTIP1;1 (accession number FJ861240) and DcTIP2; 1 (accession number FJ861241), based on similarity to Arabidopsis and maize aquaporins (Fig. 5A). DcTIP 1; 1 gene expression was unaltered by mycorrhization or by salt stress when the root was uncolonized (Fig. 5B). However, when the roots were colonized by $G$. intraradices, its expression increased wherever the salt was added (RC or HC), although the increase was higher when the salt was added to RC (Fig. 5B). In contrast, DcTIP2; 1 gene expression was downregulated by $A M$ colonization and did not change when salt stress was added to HC (Fig. 5B). Finally, DcTIP2; 1 expression rose under salt stress in noncolonized roots but was undetected when the roots were colonized (Fig. 5B).

\section{DISCUSSION}

Although the transport of water from the AM hyphae to the host roots has been described for a long time (Hardie 1985; Khalvati et al. 2005; Marualanda et al. 2003; Ruiz-Lozano and Azcón 1995), here, an aquaporin from the AM fungus $G$. intraradices, which could be involved in such transport, is reported for the first time. Based on phylogenetic analyses and on transmembrane domain analyses, the GintAQPl gene seems to be related to ER aquaporins, such as plant SIP and human AQP11 and AQP12 (Maeshima and Ishikawa 2008). GintAQP1 has a very short N-terminal tail and loop C similar to ER aquaporins, especially to plant SIP (Maeshima and Ishikawa 2008). However, contrary to ER aquaporins, GintAQP1 has conserved the two NPA motifs and has a lower predicted isoelectric point. On the other hand, GintAQP1 does not possess any diacidic motif in its N-terminal tail, similar to plant SIP (Maeshima and Ishikawa 2008), likely to favor their retention in the ER (Zelazny et al. 2009). We failed to detect any water, glycerol, or urea transport of GintAQP1 protein when it was expressed in Xenopus oocytes (data not shown). These negative results could be caused by the retention of GintAQP1 in the ER of Xenopus oocytes and, therefore, the lack of transport to the plasma membrane. This problem in detecting aquaporin activity using oocytes experiments was also reported for PIP1 plant aquaporins (Fetter et al. 2004), which are retained in ER membranes (Zelazny et al. 2009). In fact, Arabidopsis SIP1;1 and SIP1;2, and human AQP11 were de- scribed to transport water by using stopped-flow light-scattering assays of membrane vesicles from yeast cells expressing SIP (Ishikawa et al. 2005), or via proteoliposomes reconstituted with purified recombinant AQP11 (Yakata et al. 2007), avoiding the inconvenience of ER retention. Therefore, more accurate studies are needed to localize GintAQP1 and to characterize its putative transport substrates.

Based on the results of GintAQP1 gene expression analyses in roots of soil-grown plants and on the expression of aquaporins from these plants, analyzed in previous studies, it seems that the expression of GintAQP1 is coordinated with that of plant aquaporins. Therefore, in the $P$. vulgaris experiment, GintAQPI did not change its expression under salt stress, whereas the four $P$. vulgaris PIP genes analyzed increased their expression (Aroca et al. 2007). GintAQPl was upregulated under drought or cold conditions, whereas two of four $P$. vulgaris PIP genes decreased their expression and the expression of the other two remained constant (Aroca et al. 2007). These data could be interpreted as a compensatory mechanism between host plant aquaporins and the AM fungal aquaporin. In fact, in lettuce plants treated with ABA, that caused an increase of one PIP2 aquaporin gene (Aroca et al. 2008); also, a decrease on GintAQP1 gene expression was observed. However, contrary to what happened in $P$. vulgaris, lettuce plants were unable to exude any water from the roots under drought conditions, indicating a severe drought stress (Aroca et al. 2008). Therefore, this reduction in GintAQP1 gene expression could be interpreted as a water-saving mechanism (Porcel et al. 2006a). In contrast, the possible compensatory relationship between plant host aquaporins and the AM fungal one could be observed in tobacco plants. Under drought conditions, a major expression of GintAQP1 was found in the roots of antisense plants with downregulated PIP1 aquaporins. Although this compensatory mechanism could be inferred from the data of soil-grown plants, more comprehensive experiments are needed in order to confirm such a possible mechanism.

It is known that several $G$. intraradices genes are developmentally and spatially regulated, with some of them being highly expressed in the intraradical structures (Seddas et al. 2008). In the present research, GintAQP 1 was not regulated in the extraradical mycelium by any of the treatments imposed (ABA, PEG [polyethylene glycol], $\mathrm{NaCl}$, or cold). However, its expression was higher in the intraradical mycelium (considering that RNA from the RC was mainly from colonized roots and not from associated extraradical mycelium) (Recobert et al. 2009), and it was even higher after addition of $\mathrm{NaCl}$ to the

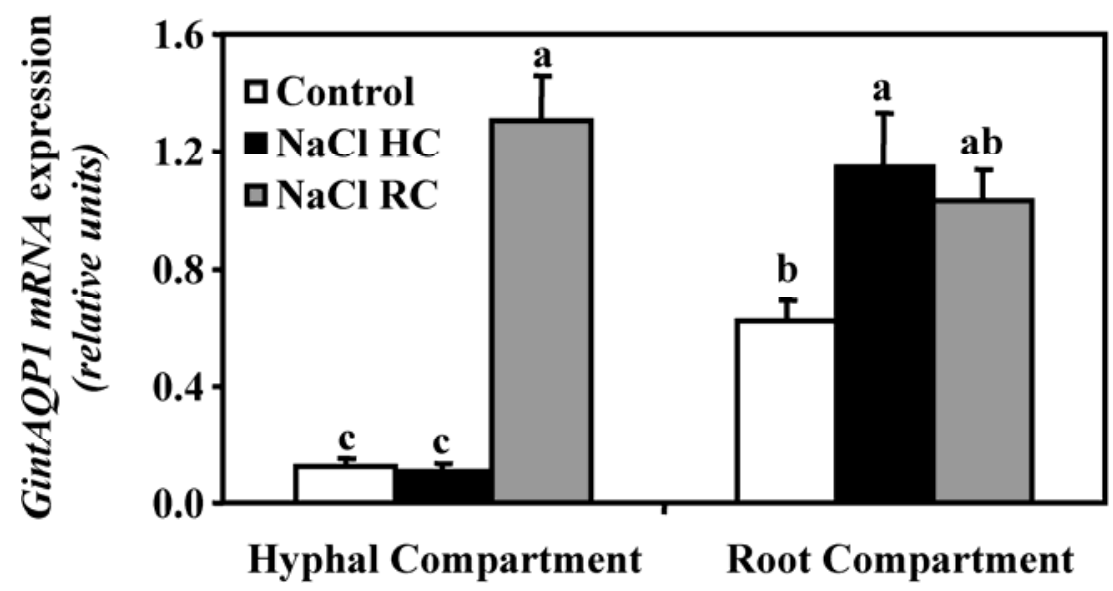

Fig. 4. GintAQP1 gene expression in arbuscular mycorrhizal fungal mycelium from the hyphal compartment (HC) or from the root compartment (RC, predominantly intraradical hyphae). Treatments are untreated (Control, white bars), $100 \mathrm{mM} \mathrm{NaCl}$ added to $\mathrm{HC}$ (black bars), or $100 \mathrm{mM} \mathrm{NaCl}$ added to RC (gray bars). Bars represent mean \pm standard error $(n=6)$. Bars with the same letter indicate non significant differences $(P>0.05)$ after analysis of variance and least significant difference tests. 
HC. These results show that GintAQP1 was upregulated by exposure of the extraradical mycelium to an osmotic stress, although the intraradical mycelium was not subjected to $\mathrm{NaCl}$ stress. This upregulation of GintAQPl in the RC could be related to the transport of water from the isotonic medium (RC) to the hypertonic one (HC), contributing to alleviate the osmotic stress of the HC mycelium. This hypothesis was supported by the fact that GintAQP1 expression also increased in the $\mathrm{HC}$ (now the isotonic medium) mycelium when $\mathrm{NaCl}$ was applied to the RC (now the hypertonic medium), while nonsignificant changes in its expression were observed in the RC. Therefore, it is likely to presume a cross-talk or communication between the fungal mycelium growing in the two plate compartments when an osmotic stress was applied to one of the compartments. These results showed regulation of an AM fungal gene expression in one fungal structure not subjected to any stress, caused by environmental changes affecting another fungal structure. The different signaling mechanisms carrying out this communication from the stressed fungal structure to the nonstressed one may be of a hormonal nature, like ABA (Herrera-Medina et al. 2007); hydraulic nature (Christmann et al. 2007); or electrical nature (Zimmermann et al. 2009). Besides the possible production of the hormone ABA by the AM fungal partner (Esch et al. 1994), no evidences of hydraulic or electrical signaling mechanisms have been described thus far in AM fungi. However, it is known that phytopathogenic fungi alter the membrane electrical potential gradient in the host tissues (Felle et al. 2008). How electric potential gradients could be transmitted along the AM hyphae remains unknown.

It is known that AM symbiosis alters the aquaporin expression pattern of the host roots (Aroca et al. 2007; Ouziad et al. 2006; Porcel et al. 2006a; Ruiz-Lozano and Aroca 2008). So, we were interested in knowing whether the communication observed between the AM mycelia from both plate compartments also was valid for the host carrot aquaporins. The two TIP aquaporins cloned from $D$. carota cDNA roots were regulated differently by the AM symbiosis. DcTIP2; 1 was downregulated by the symbiosis, and even more when $\mathrm{NaCl}$ was applied to the RC, where no gene expression was detected, in contrast to noncolonized roots, where a significant increase was observed. An opposite trend was observed for DcTIP1;1, which only increased its expression under salt conditions when roots were colonized. These results agree with those in the literature because each aquaporin gene may respond specifically to a given stress and also depending on the presence or absence of the AM symbiont (Aroca et al. 2007). However, the most inter-

Fig. 5. A, Phylogenetic tree of Arabidopsis, maize and, carrot tonoplast intrinsic proteins (TIPs) based on neighbor-joining method. DcTIP1;1 and DcTIP2;1 are underlined. The abbreviations for species names are At (Arabidopsis thaliana), Zm (Zea mays), and Dc (Daucus carota). The accession numbers of the amino acid sequences used are AtTIP1-1 (AAD31569), AtTIP1-2 (BAB01832), AtTIP1-3 (AAC62778), AtTIP2-1 (BAB01264), AtTIP2-2 (CAB10515), AtTIP2-3 (BAB09071), AtTIP3-1 (AAG52132), AtTIP3-2 (AAF97261), AtTIP4-1 (AAC42249), AtTIP5-1 (CAB51216), ZmTIP1-1 (AF037061), ZmTIP1-2 (AF326500), ZmTIP2-1 (AF326501), ZmTIP2-2 (AF326502), ZmTIP2-3 (AF326503), ZmTIP3-1 (AF326504), ZmTIP4-1 (AF326505), ZmTIP4-2 (AF326506), ZmTIP4-3 (AF326507), ZmTIP4-4 (AF326508), ZmTIP5-1 (AF326509), DcTIP1-1 (FJ861240), and DcTIP2-1 (FJ861241). B, DcTIP1;1 (upper panel) and DcTIP2;1 (lower panel) gene expression in carrot roots grown in vitro (from left to right) untreated and uncolonized by Glomus intraradices, untreated and colonized by $G$. intraradices, uncolonized and treated with 100 $\mathrm{mM} \mathrm{NaCl}$ in the root compartment (RC) for 1 week, colonized and treated with $100 \mathrm{mM} \mathrm{NaCl}$ in the RC for 1 week, and colonized and harvested after 1 week of adding $100 \mathrm{mM} \mathrm{NaCl}$ to the hyphal compartment. Bars with the same letter indicate nonsignificant differences $(P>0.05)$ after analysis of variance and least significant difference tests; n.d. $=$ not detected. esting result was that DcTIP1;1 gene expression also was upregulated when $\mathrm{NaCl}$ was applied to $\mathrm{HC}$. This result supports the possible communication between the two parts of the AM mycelium subjected to different environmental conditions, regulating also the gene expression of the host roots not exposed directly to the stressful conditions. As far as we know, this is the first report where this kind of communication has been described at the gene level. The upregulation of GintAQPI and $D c T I P 1 ; 1$ upon exposure of $\mathrm{HC}$ to $\mathrm{NaCl}$ or the upregulation of GintAQPl in the $\mathrm{HC}$ when $\mathrm{NaCl}$ was applied to $\mathrm{RC}$ could be interpreted as a mechanism of transporting water from an iso-osmotic medium to a hyperosmotic one through the AM mycelium. In fact, Egerton-Warburton and associates (2007) found water transport from well-watered plants to
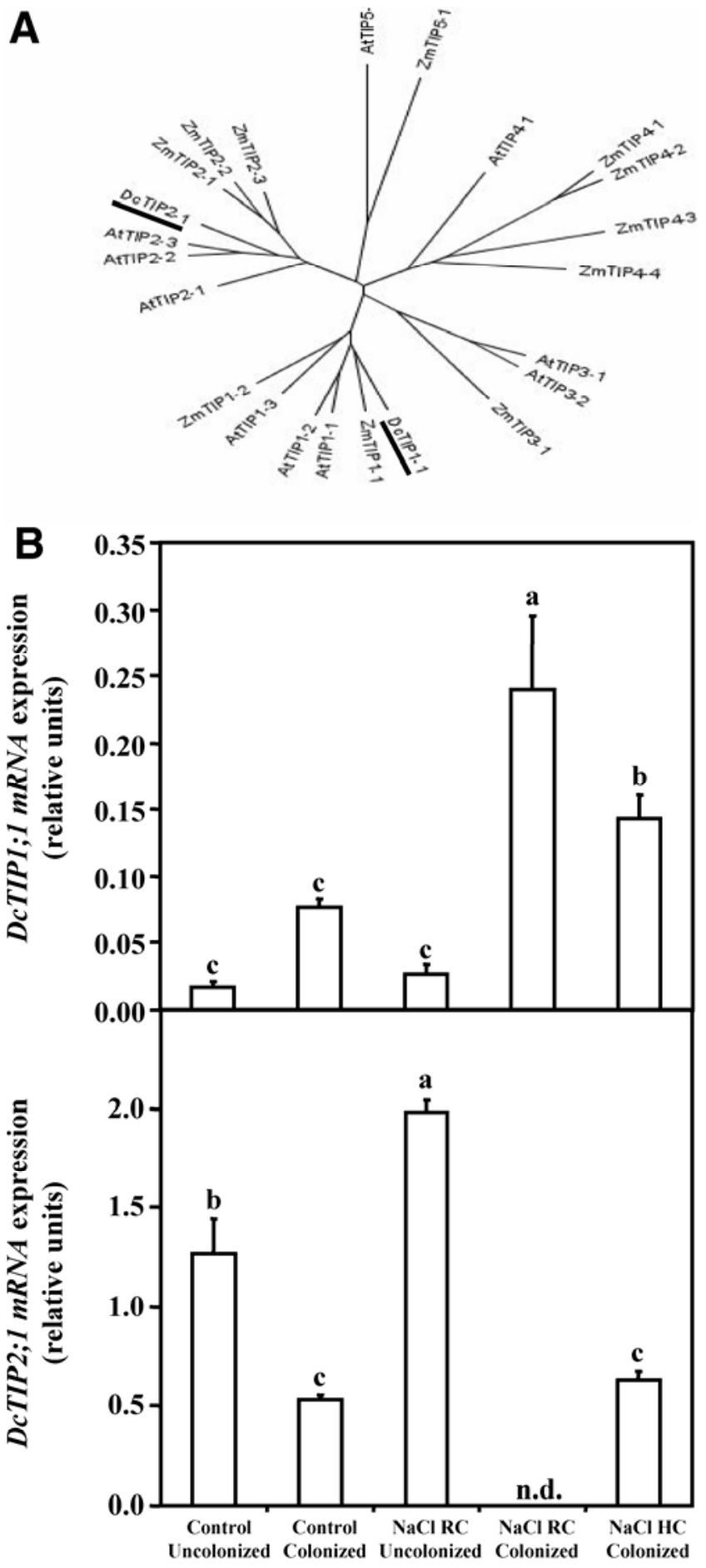
plants subjected to drought through ectomycorrhizal and AM hyphae, increasing the transport rate as the drought was more severe.

In addition to the first description of an aquaporin gene from an AM fungus, the main findings of the present research could be summarized as follows. i) GintAQP1 expression responded differently to each environmental stress and host plant, although a certain compensatory mechanism between the host aquaporins and GintAQP1 expressions could be inferred. ii) GintAQP1 increased its expression in the unstressed part of the mycelium when the other part was subjected to an osmotic stress by $\mathrm{NaCl}$, implicating a communication between the two parts of the mycelium that allows a spatial regulation of gene expression. iii) Similarly, the host aquaporin DcTIP1; 1 also increased its expression when only the extraradical mycelium from $\mathrm{HC}$ was treated with $\mathrm{NaCl}$, also supporting a communication mechanism involving the extra and intraradical mycelium and the host root. Further studies are needed to determine precisely where the GintAQP1 is localized inside the mycelium and what its putative transport substrates could be.

\section{MATERIALS AND METHODS}

\section{Cloning of $G$. intraradices aquaporin gene.}

G. intraradices was established in a monoxenic culture (St.Arnaud et al. 1996), as described by Porcel and associates (2006b) and Jahromi and associates (2008). Briefly, clone DC2 of carrot (D. carota L.) Ri-T DNA-transformed roots were cultured with the AM fungus $G$. intraradices Smith and Shenk (DAOM 197198, Biosystematic Research Center, Ottawa, Canada) in two-compartment petri dishes. Culture was initiated in one compartment (RC) of each plate, which contained minimal medium. Fungal hyphae but not roots were allowed to grow over to the second compartment (HC), which contained liquid minimal medium without sucrose (M-C medium). The plates were incubated in the dark at $24^{\circ} \mathrm{C}$ for 3 months. Fungal mycelium was carefully removed from the $\mathrm{HC}$, and RNA was extracted by using the RNesay plant mini kit (Qiagen, Valencia, CA, U.S.A.). cDNA was synthesized from such RNA as previously described by Porcel and associates (2006a). Synthesized DNA was amplified by PCR using degenerate primers designed from alignment of different plant and fungal aquaporins: DEGAQPFor, 5'-GG(A/T)GG(C/T)(A/T)(A/T) TTIAA (C/T)CC(A/T) GC(A/C/T)GT-3' and DEGAQPRev, 5'-CC(A/ $\mathrm{C} / \mathrm{T}) \mathrm{A}(\mathrm{A} / \mathrm{G})(\mathrm{A} / \mathrm{G}) \mathrm{GA}(\mathrm{C} / \mathrm{T}) \mathrm{C}(\mathrm{G} / \mathrm{T})(\mathrm{A} / \mathrm{T}) \mathrm{GC}(\mathrm{A} / \mathrm{T}) \mathrm{GG}(\mathrm{A} / \mathrm{G}) \mathrm{TT}-$ 3'. The PCR program was as described by Aroca and associates (2006). Amplified products were separated in $2 \%$ agarose gel, and visible bands of the expected size (approximately 400 $\mathrm{kb}$ ) were eluted with QIAquick gel extraction kit (Qiagen) and cloned into pGEM plasmid (Promega Corp., Madison, WI, U.S.A.) and used to transform Escherichia coli DH-5 $\alpha$-competent cells. The plasmid DNA was purified and sequenced, which allowed identification of a clone with a putative aquaporin sequence.

For the determination of the full-length sequence of the obtained cDNA, 5' - and 3'-RACE technique was carried out on total RNA using the SMART RACE cDNA Amplification Kit (Clontech, Mountain View, CA, U.S.A.). After 5'- and 3'RACE, new primers were designed covering the full gene and PCR was performed with high-fidelity $P f u$ DNA polymerase (Stratagene, La Jolla, CA, U.S.A.).

The alignment of GintAQP1 with other plant, human, and fungal aquaporins was carried out by ClustalW XXL method. The phylogenetic tree was obtained by using Treelllustrator VO.52 Beta using the neighbor-joining method. The hypothetical plasma membrane domains of GintAQP1 were predicted by the SOSUI program (Hirokawa et al. 1998).
Later, and based on GintAQP1 expression analyses, two different carrot aquaporins were cloned and identified following the same procedure as described above. The original RNA was isolated from the same carrot roots used above but grown without any AM fungus.

\section{Southern blot.}

Southern blot was carried out as described by Ruiz-Lozano and Bonfante (2000). Briefly, specific primers flanking the full open reading frame (ORF) of the GintAQP1 were designed (forward [For], ATGGGATTAAAGGATGACTTTGTG and reverse [Rev], TTAATCCTTACCAAGATTTTCCCTA), and PCR at an annealing temperature of $56^{\circ} \mathrm{C}$ was carried out with genomic DNA extracted from spores and mycelium of $G$. intraradices and from $D$. carota roots. PCR products were transferred to Hybond- $\mathrm{N}^{+}$nylon membrane by capillarity; DNA was denatured and fixed by exposition to UV light. The DNA corresponding to the full ORF was labeled by a chemiluminiscent detection kit (ECL direct DNA labeling and detection system, Amersham, U.K.) and hybridized with the membrane overnight at $42^{\circ} \mathrm{C}$ with the buffer supplied by the kit. After washing, an X-ray film was exposed to the membrane and developed. Genomic DNAs were isolated as described previously by Ruiz-Lozano and Bonfante (2000).

\section{GintAQP1 transport activity assays.}

Analysis of GintAQP1 water transport activity was carried out by expressing the ORF of the gene in Xenopus laevis oocytes as previously described by Mut and associates (2008). Briefly, the full-length ORF of GintAQP1 and AtPIP2;3 used as a positive control (Daniels et al. 1994) were cloned into pSP64T-derived vector carrying 5' and 3' untranslated sequences of a $\beta$-globin gene from X. laevis (Agre et al. 1999) and complementary RNAs were synthesized (Mut et al. 2008). After 3 days of cRNA or distilled water injection, mature $X$. laevis oocytes were transferred to a hypotonic medium and their osmotic water permeability coefficient was calculated as described by Agre and associates (1999). Measurements of glycerol and urea transport activities were carried out in X. laevis oocytes as previously described by Soto and associates (2008).

\section{Biological material.}

Two different kinds of experiments were carried out. First, the expression of GintAQP1 was analyzed in colonized roots of $P$. vulgaris, L. sativa, or $N$. tabacum plants. Plants growing in pots filled with a mixture of sterilized soil and sand (1:1) were inoculated with the AM fungus $G$. intraradices isolate BEG 123 (Aroca et al. 2007, 2008; Porcel et al. 2005). P. vulgaris plants were subjected to cold, drought, or salt stress as previously described by Aroca and associates (2007). L. sativa plants were subjected to drought and supplied with exogenous ABA as described by Aroca and associates (2008). Two N. tabacum plants lines, wild-type or NtAQP1 antisense line (Siefritz et al. 2002), were subjected to drought as described by Porcel and associates (2005). At harvest, roots were carefully cleaned with tap water before being stored at $-80^{\circ} \mathrm{C}$ in order to avoid contaminations with extraradical mycelium. Total root RNA from different plants was extracted by a phenol/chloroform method followed by precipitation with $\mathrm{LiCl}$ (Kay et al. 1987), and cDNAs were synthesized as described by Aroca and associates (2007).

The second set of experiments were carried out in vitro, with the AM fungus $G$. intraradices growing in monoxenic cultures (Jahromi et al. 2008; Porcel et al. 2006b), or with uninoculated carrot roots growing under the same conditions but in absence of the fungus (see above). Three different treatments were applied to the $\mathrm{HC}$ and one to the whole plate. Water stress 
was imposed to HC by adding 25\% of PEG 6000 (SigmaAldrich Co., Madrid, Spain) to the liquid minimal medium, corresponding to a water potential of $-1.5 \mathrm{MPa}$. The mycelium grew under this condition for 5 days (Porcel et al. 2006b). Salt stress was imposed to $\mathrm{HC}$ by applying a final concentration of $100 \mathrm{mM} \mathrm{NaCl}$; the mycelium was grown under this condition for 1 week. The $\mathrm{NaCl}$ concentration was selected based on the study by Jahromi and associates (2008), because it was the lowest concentration with a significant effect on hyphal growth and spore production. Exogenous ABA was applied to the $\mathrm{HC}$ at a final concentration of $100 \mu \mathrm{M}$ and the mycelium was grown there for 1 day. Finally, the whole plates were kept at $5^{\circ} \mathrm{C}$ in the dark for 3 days. At the end of all treatments, untreated and treated mycelia from $\mathrm{HC}$ were isolated, immersed in liquid $\mathrm{N}_{2}$, and stored at $-80^{\circ} \mathrm{C}$ until RNA was extracted by the RNesay plant mini kit (Qiagen). In a separate set of experiments, carrot roots colonized or not with the AM fungus were subjected to a final concentration of $100 \mathrm{mM} \mathrm{NaCl}$ for 1 week. Roots were removed from the medium and hyphal debris were carefully removed with forceps before they were immersed in liquid $\mathrm{N}_{2}$ and stored at $-80^{\circ} \mathrm{C}$. Total root RNA was extracted as described for the mycelium protocol.

\section{Gene expression analyses.}

GintAQP1 and carrot aquaporin DcTIP1;1 and DcTIP2;1 gene expression analyses were carried out by quantitative reverse-transcription (qRT)-PCR using iCycler (BioRad, Hercules, CA, U.S.A.), as described by Porcel and associates (2006b), with an annealing temperature of $57^{\circ} \mathrm{C}$. cDNA was synthesized as described above from the RNA samples. Each reaction mixture of $25 \mu \mathrm{l}$ contained $1 \mu \mathrm{l}$ of a 1:10 dilution of the corresponding cDNA, $200 \mathrm{nM}$ dNTPs, $400 \mathrm{nM}$ each primer, $3 \mathrm{mM}$ $\mathrm{MgCl}_{2}, 2.5 \mu \mathrm{l}$ of $1 \times \mathrm{SYBR}$ Green (Molecular Probes, Eugene, OR, U.S.A.), and $0.5 \mathrm{U}$ of Platinum Taq DNA polymerase (Invitrogen, Carlsbad, CA, U.S.A.) in $1 \times$ PCR buffer $(20 \mathrm{mM}$ Tris- $\mathrm{HCl}, \mathrm{pH} 8.4$, and $50 \mathrm{mM} \mathrm{KCl}$ ). The primers used in the qRT-PCR experiments were as follows: for GintAQP1, For, 5'AGGACTCGGAGGTAGTGATGC-3' and Rev, 5'-GCCGGAT ATATCACTCCAAAGC-3'; for Gint18S rRNA, For, 5'-TGTT AATAAAAATCGGTGCGTTGC-3' and Rev, 5'-AAAACGC AAATGATCAACCGGAC-3' (Gónzalez-Guerrero et al. 2005; Porcel et al. 2006b); for DcTIP1;1, For, 5'-GTGGCTGTCGG AGCTAATATC-3' and Rev, 5'-CAGTGGAGCCAAGACACT GAG-3'; for DcTIP2; 1, For, 5'-GGATCCTGCTGGATTAGTA GC-3' and Rev, 5'-TGTTGGCTGCTATGGACACTC-3'; and for carrot actin gene, For, 5'-GTGACGTTGATATCAGAAA GG-3' and Rev, 5'-CGTCATATTCACCCTTCGAG-3' (Kihara et al. 2003). Experiments were repeated three times using cDNAs originated from two RNAs extracted from two different biological samples.

\section{ACKNOWLEDGMENTS}

The work was supported by a grant from Ministerio de Ciencia e Inovación, Spain (project AGL-2008-00898).

\section{LITERATURE CITED}

Agre, P., Mathai, J. C., Smith, B. L., and Preston, G. M. 1999. Functional analyses of aquaporin water channel proteins. Pages 550-572 in: Methods in Enzymology. P. M. Conn, ed. Academic Press, San Diego, CA, U.S.A.

Aharon, R., Shahak, Y., Wininger, S., Bendov, R., Kapulnik, Y., and Galili, G. 2003. Overexpression of a plasma membrane aquaporin in transgenic tobacco improves plant vigor under favorable growth conditions but not under drought or salt stress. Plant Cell 15:439-447.

Allen, J. W., and Shachar-Hill, Y. 2009. Sulfur transfer through an arbuscular mycorrhiza. Plant Physiol. 149:549-560.

Aroca, R., Ferrante, A., Vernieri, P., and Chrispeels, M. J. 2006. Drought, abscisic acid, and transpiration rate effects on the regulation of PIP aquaporin gene expression and abundance in Phaseolus vulgaris plants. Ann. Bot. 98:1301-1310.

Aroca, R., Porcel, R., and Ruiz-Lozano, J. M. 2007. How does arbuscular mycorrhizal symbiosis regulate root hydraulic properties and plasma membrane aquaporins in Phaseolus vulgaris under drought, cold, or salinity stresses? New Phytol. 173:808-816.

Aroca, R., Vernieri, P., and Ruiz-Lozano, J. M. 2008. Mycorrhizal and non-mycorrhizal Lactuca sativa plants exhibit contrasting responses to exogenous ABA during drought stress and recovery. J. Exp. Bot. 59:2029-2041.

Augé, R. M. 2001. Water relations, drought and vesicular-arbuscular mycorrhizal symbiosis. Mycorrhiza 11:3-42.

Bonfante, P., and Genre, A. 2008. Plants and arbuscular mycorrhizal fungi: An evolutionary-developmental perspective. Trends Plant Sci. 13:492498.

Chrispeels, M. J., and Agre, P. 1994. Aquaporins-water channel proteins of plant and animal cells. Trends Biochem. Sci. 19:421-425.

Christmann, A., Weilier, E.W., Steudle, E., and Grill, E. 2007. A hydraulic signal in root-to-shoot signaling of water shortage. Plant J. 52:167-174.

Daniels, M. J., Mirkov, T. E., and Chrispeels, M. J. 1994. The plasma membrane of Arabidopsis thaliana contains a mercury-insensitive aquaporin that is homologue of the tonoplast water channel TIP. Plant Physiol. 106:1325-1333.

Egerton-Warburton, L. M., Querejeta, J. I., and Allen, M. F. 2007. Common mycorrhizal networks provide a potential pathway for the transfer of hydraulically lifted water between plants. J. Exp. Bot. 58:1473-1483.

Esch, H., Hundeshagen, B., Schneiderpoetsch, H., and Bothe, H. 1994. Demonstration of abscisic-acid in spores and hyphae of the arbuscularmycorrhizal fungus Glomus and in the $\mathrm{N}_{2}$-fixing cyanobacterium Anabaena-variabilis. Plant Sci. 99:9-16.

Felle, H. H., Herrmann, A., Shäfer, P., Hückelhoven, R., and Kogel, K.-H. 2008. Interactive signal transfer between host and pathogen during successful infection of barley leaves by Blumeria graminis and Bipolaris sorokiniana. J. Plant Physiol. 165:52-59.

Fetter, K., Van Wilder, V., Moshelion, M., and Chaumont, F. 2004. Interactions between plasma membrane aquaporins modulate their water channel activity. Plant Cell 16:215-228.

Forrest K. L., and Bhave, M. 2007. Major intrinsic proteins (MIPs) in plants: A complex gene family with major impacts on plant phenotype. Funct. Integr. Genomics 7:263-289.

Gónzalez-Guerrero, M., Azcón-Aguilar, C., Mooney, M., Valderas, A., MacDiarmid, C. W., Eide, D. J., and Ferrol, N. 2005. Characterization of a Glomus intraradices gene encoding a putative $\mathrm{Zn}$ transporter of the cation diffusion facilitator family. Fungal Genet. Biol. 42:130-140.

Hardie, K. 1985. The effect of removal extraradical hyphae on wateruptake by vesicular arbuscular mycorrhizal plants. New Phytol. 101:677-684.

Hartung, W., Schraut, D., and Jiang, F. 2005. Physiology of abscisic acid (ABA) in roots under stress - a review of the relationship between root ABA and radial water and ABA flows. Aust. J. Agric. Res. 56:12531259.

Herrera-Medina, M. J., Steinkellner, S., Vierheilig, H., Ocampo, J. A., and García-Garrido, J. M. 2007. Abscisic acid determines arbuscule development and functionality in the tomato arbuscular mycorrhiza. New Phytol. 175:554-564.

Hirokawa, T., Boon-Chieng, S., and Mitaku, S. 1998. SOSUI: Classification and secondary structure prediction system for membrane proteins. Bioinformatics 14:378-379.

Ishikawa F., Suga, S., Uemura, T., Sato, M. H., and Maeshima, M. 2005. Novel type aquaporin SIPs are mainly localized to the ER membrane and show cell-specific expression in Arabidopsis thaliana. FEBS (Fed. Eur. Biochem. Soc.) Lett. 579:5814-5820.

Jahromi, F., Aroca, R., Porcel, R., and Ruiz-Lozano, J. M. 2008. Influence of salinity on the in vitro development of Glomus intraradices and on the in vivo physiological and molecular responses of mycorrhizal lettuce plants. Microb. Ecol. 55:45-53.

Jang, J. Y., Lee, S. H., Ree, J. Y., Chung, G. C., Ahn, S. J., and Kang, H. 2007. Transgenic Arabidopsis and tobacco plants overexpressing and aquaporin respond differently to various abiotic stresses. Plant Mol. Biol. 64:621-632.

Katsuhara, M., Koshio, K., Shibasaka, M., Hayashi, Y., Hayakawa, T., and Kasamo, K. 2003. Over-expression of a barley aquaporin increased the shoot/root ratio and raised salt sensitivity in transgenic rice plants. Plant Cell Physiol. 44:1378-1383.

Kay, R., Chau, A., and Daly, M. 1987. Duplication of CaMV 35S promoter sequences creates a strong enhancer for plant genes. Science 236:12991302.

Khalvati, M. A., Hu, Y., Mozafar, A., and Schmidhalter, U. 2005. Quantification of water uptake by arbuscular mycorrhizal hyphae and its signifi- 
cance for leaf growth, water relations, and gas exchange of barley subjected to drought stress. Plant Biol. 7:706-712.

Kihara, T., Ohno, T., Koyama, H., Sawafuji, T., and Hara, T. 2003. Characterization of NADP-isocitrate dehydrogenase expression in a carrot mutant cell line with enhanced citrate excretion. Plant Soil 248:145-153.

Koide, R. T., and Mosse, B. 2004. A history of research on arbuscular mycorrhiza. Mycorrhiza 14:145-163.

Krajinski, F., Biela, A., Schubert, D., Gianinazzi-Pearson, V., Kaldenhoff, R., and Franken, P. 2000. Arbuscular mycorrhiza development regulates the mRNA abundance of Mtaqpl encoding a mercury-insensitive aquaporin of Medicago truncatula. Planta 211:85-90.

Laizé, V., Tacnet, F., Ripoche, P., and Hohmann, S. 2000. Polymorphism of Saccharomyces cerevisiae aquaporins. Yeast 16:897-903.

Lian H.-M., Yu, X., Ye, Q., Ding, X.-S., Kitagawa, Y., Kwak, S.-S., Su, W.A., and Tang, Z.-C. 2004. the role of aquaporin RWC3 in drought avoidance in rice. Plant Cell Physiol. 45:481-489.

Maeshima, M., and Ishikawa, F. 2008. ER membrane aquaporins in plants Pfluegers Arch. 456:709-716.

Marulanda, A., Azcón, R., and Ruiz-Lozano, J. M. 2003. Contribution of six arbuscular mycorrhizal fungal isolates to water uptake by Lactuca sativa plants under drought stress. Physiol. Plant. 119:526-533.

Matsuo, N., Ozawa, K., and Mochizuki, T. 2009. Genotypic differences in root hydraulic conductance of rice (Oryza sativa L.) in response to water regimes. Plant Soil 316:25-34

Maurel, C., Verdoucq, L., Luu, D.-T., and Santoni, V. 2008. Plant aquaporins: Membrane channels with multiple integrated functions. Annu. Rev. Plant Biol. 59:595-624.

Muhsin, T. M., and Zwiazek, J. J. 2002. Colonization with Hebeloma crustuliniforme increases water conductance and limits shoot sodium uptake in white spruce (Picea glauca) seedlings. Plant Soil 238:217-225.

Mut, P., Bustamante, C., Martínez, G., Alleva, K., Sutka, M., Civello, M., and Amodeo, G. 2008. A fruit-specific plasma membrane aquaporin subtype PIP1;1 is regulating during strawberry (Fragaria $\times$ ananassa) fruit ripening. Physiol. Plant. 132:538-551.

Oliveira, R., Lages, F., Silva-Graca, M., and Lucas, C. 2003. Fps1p channel is the mediator of the major part of glycerol passive diffusion in Saccharomyces cerevisiae: Artefacts and re-definitions. Biochim. Biophys. Acta. 1613:57-71

Ouziad, F., Wilde, P., Schmelzer, E., Hildebrandt, U., and Bothe, H. 2006. Analysis of expression of aquaporins and $\mathrm{Na}^{+} / \mathrm{H}^{+}$transporters in tomato colonized by arbuscular mycorrhizal fungi and affected by salt stress. Environ. Exp. Bot. 57:177-186.

Peng, Y., Lin, W., Cai, W., and Arora, R. 2007. Overexpression of a Panax ginseng tonoplast aquaporin alters salt tolerance, drought tolerance and cold acclimation ability in transgenic Arabidopsis plants. Planta 226:729-740

Pettersson, N., Filipsson, C., Becit, E., Brive, L., and Hohmann, S. 2005. Aquaporins in yeasts and filamentous fungi. Biol. Cell 97:487-500.

Pirozynski, K. A., and Malloch, D. W. 1975. The origin of land plants: A matter of mycotropism. Biosystems 6:153-164

Porcel, R., Gómez, M., Kaldenhoff, R., and Ruiz-Lozano, J. M. 2005. Impairment of NtAQP1 gene expression in tobacco plants does not affect root colonization pattern by arbuscular mycorrhizal fungi but decreases their symbiotic efficiency under drought. Mycorrhiza 15:417-423.

Porcel, R., Aroca, R., Azcón. R., and Ruiz-Lozano, J. M. 2006a. PIP aquaporin gene expression in arbuscular mycorrhizal Glycine max and Lactuca sativa plants in relation to drought stress tolerance. Plant Mol. Biol. 60:389-404.

Porcel, R., Aroca, R., Cano, C., Bago, A., and Ruiz-Lozano J. M. 2006b. Identification of a gene from the arbuscular mycorrhizal fungus Glomus intraradices encoding for a 14-3-3 protein that is up-regulated by drought stress under AM symbiosis. Microb. Ecol. 52:575-582.

Recorbert, G., Rogniaux, H., Gianinazzi-Pearson, V., and Dumas-Gaudot, E. 2009. Fungal proteins in the extra-radical phase of arbuscular mycorrhiza: A shotgun proteomic picture. New Phytol. 181:248-260.

Rojek, A., Praetorious, J., Frokiaer, J., Nielsen, S., and Fenton, R. A. 2008. A current view of mammalian aquaglyceroporins. Annu. Rev. Physiol. 70:301-327.

Rosendahl, S. 2008. Communities, populations and individuals of arbuscular mycorrhizal fungi. New Phytol. 178:253-266.

Roussel, H., Bruns, S., Gianinazzi-Pearson, V., Hahlbrock, K., and Franken, P. 1997. Induction of a membrane intrinsic protein-encoding
mRNA in arbuscular mycorrhiza and elicitor-stimulated cell suspension cultures of parsley. Plant Sci. 126:203-210.

Ruiz-Lozano, J. M. 2003. Arbuscular mycorrhizal symbiosis and alleviation of osmotic stress. New perspectives for molecular studies. Mycorrhiza 13:309-317.

Ruiz-Lozano, J. M., and Aroca, R. 2008. Last insights into the role of aquaporins in the alleviation of osmotic stress by arbuscular mycorrhizal symbiosis. Pages 139-154 in: Microbial Ecology Research Trends. T. Van Dijk, ed. Nova Science Publishers, Inc., New York.

Ruiz-Lozano, J. M., and Azcón, R. 1995. Hyphal contribution to water uptake in mycorrhizal plants as affected by fungal species and water status. Physiol. Plant. 95:472-478.

Ruiz-Lozano, J. M., and Bonfante, R. 2000. A Burkholderia strain living inside the arbuscular mycorrhizal fungus Gigaspora margarita posses the vacB gene, which is involved in host cell colonization by bacteria. Microb. Ecol. 39:137-144.

Ruiz-Lozano, J. M., Porcel, R., and Aroca, R. 2006. Does the enhanced tolerance of arbuscular mycorrhizal plants to water deficit involve modulation of drought-induced plant genes? New Phytol. 171:693-698.

Sade, N., Vinocur, B. J., Diber, A., Shatil, A., Ronen, G., Nissan, H., Wallach, R., Karchi, H., and Moshelion, M. 2009. Improving plant stress tolerance and yield production: Is the tonoplast aquaporin SITIP2;2 a key to isohydric to anisohydric conversion? New Phytol. 181:651-661

Seddas, P. M. A., Arnould, C., Tollot, M., Arias C. M., and GianinazziPearson, V. 2008. Spatial monitoring of gene activity in extraradical and intraradical stages of arbuscular mycorrhizal fungi by direct fluorescent in situ RT-PCR. Fungal Genet. Biol. 45:1155-1165.

Siefritz, F., Tyree, M. T., Lovisolo, C., Schubert, A., and Kaldenhoff, R. 2002. PIP1 plasma membrane aquaporins in tobacco: From cellular effects to function in plants. Plant Cell 14:869-876.

Soto, G., Alleva, K., Mazzella, M. A., Amodeo, G., and Muschieti, J. P. 2008. AtTIP1;3 and AtTIP5;1, the only highly expressed Arabidopsis pollen-specific aquaporins, transport water and urea. FEBS (Fed. Eur. Biochem. Soc.) Lett. 582:4077-4082.

St.-Arnaud, M., Hamel, C., Vimard, B., Caron, M., and Fortin, J. A. 1996. Enhanced hyphal growth and spore production of the arbuscular mycorrhizal fungus Glomus intraradices in an in vitro system in the absence of host roots. Mycol. Res. 100:328-332.

Uehlein, N., Fileschi, K., Eckert, M., Bienert, G. P., Bertl, A., and Kaldenhoff, R. 2007. Arbuscular mycorrhizal symbiosis and plant aquaporins expression. Phytochemistry 68:122-129.

Van Aelst, L., Hohmann, S., Zimmermann, F. K., Jans, A. W. H., and Thevelein, J. M. 1991. A yeast homologue of the bovine lens fibre MIP gene family complements the growth defect of a Saccharomyces cerevisiae mutant on fermentable sugars but not its defect in glucoseinduced RAS-mediated cAMP siganalling. EMBO (Eur. Mol. Biol. Organ.) J. 10:2095-2104.

Wallace, I. S., Choi, W. G., and Roberts, D. M. 2006. The structure, function and regulation of the nodulin 26-like intrinsic protein family of plant aquaglyceroporins. Biochim. Biophys. Acta. 1758:1165-1175.

Yakata, K., Hirokai, Y., Ishibashi, K., Sohara, E., Sasaki, S., Mitsuoka, K. and Fujiyoshi, Y. 2007. Aquaporin-11 containing divergent NPA motif has normal water channel activity. Biochim. Biophys. Acta 1768:688 693.

Yu, Q., Hu, Y., Li, J., Wu, Q., and Lin, Z. 2005. Sense and antisense expression of plasma membrane aquaporin BnPIPl from Brassica napus in tobacco and its effects on plant drought resistance. Plant Sci. 169:647-656.

Zelazny, E., Miecielica, U., Borst, J.W., Hemminga, M. A., and Chaumont, F. 2009. An N-terminal diacidic motif is required for the trafficking of maize aquaporins ZmPIP2;4 and ZmPIP2;5 to the plasma membrane. Plant J. 57:346-355.

Zimmermann, M. R., Maischack, H., Mithöfer, A., Boland, W., and Felle, H. H. 2009. System potentials, a novel electrical long-distance apoplastic signal in plants, induced by wounding. Plant Physiol. 149:15931600

\section{AUTHOR-RECOMMENDED INTERNET RESOURCE}

ClustalW XXL: www.ch.embnet.org/software/ClustalW-XXL.html 